\title{
Las revistas profesionales especializadas en publicidad en España: resultados de un estudio Delphi
}

\author{
Antonio J. Baladrón Pazos \\ Profesor de Publicidad y Relaciones Públicas. Universidad Rey Juan Carlos \\ Beatriz Correyero Ruiz \\ Profesora de Periodismo. Universidad Católica San Antonio
}

Resumen:

En este artículo se recogen los resultados de una investigación cualitativa desarrollada en 2007 sobre el mercado de las revistas profesionales especializadas en publicidad en España. El objetivo principal fue llevar a cabo un análisis descriptivo de la situación actual y complementarlo con un análisis prospectivo con el fin de prever el futuro de estas publicaciones. La técnica de investigación utilizada fue el método Delphi. Las conclusiones se refieren a cuestiones como las fortalezas y debilidades de estas revistas, sus principales competidores, el uso que hacen de las nuevas tecnologías o las tendencias de futuro.

Palabras clave:

Publicidad, revistas profesionales, negocio editorial, tendencias, investigación cualitativa, método Delphi Abstract:

In this article there are gathered the results of a qualitative research developed in 2007 on the market of the professional magazines specialized in advertising in Spain. The main purpose was to carry out a descriptive analysis of the current situation and also a prospective analysis in order to foresee the future of these publications. The research methodology used was the Delphi method. The conclusions refer to questions like the strengths and weaknesses of these magazines, their principal competitors, how they use the new technologies and the future trends related to their development.

Keywords:

Advertising, professional magazines, publishing business, tendencies, qualitative investigation, Delphi method 


\section{Introducción: objeto de estudio y objetivo}

El objeto de estudio de la investigación que aquí se presenta son las revistas profesionales especializadas en publicidad en España (en adelante, RPEPE). Se trata de un mercado bastante estable, que despuntó en las dos últimas décadas del siglo XX y que en la actualidad cuenta con un número de títulos prácticamente constante y una cuota de mercado limitada por su naturaleza especializada. No obstante, a pesar de esa estabilidad, los cambios producidos en los últimos años en el mercado mediático, derivados de la creciente aceptación social de las nuevas tecnologías de la información y la comunicación, o la propia evolución del sector publicitario, sitúan a estas cabeceras ante una situación de necesaria mejora continua. Con este estudio hemos querido aportar tanto un análisis descriptivo del panorama general de las RPEPE como un análisis prospectivo a través del cual evidenciar algunas de las principales tendencias de futuro para este mercado.

En el siguiente epígrafe de este artículo se exponen algunas aclaraciones metodológicas que creemos necesario hacer constar para justificar la elección del método Delphi como técnica de investigación utilizada en este trabajo. A continuación, en el tercer epígrafe, se detallan los aspectos básicos del diseño de la investigación. El cuarto apartado se ha reservado para presentar los resultados del estudio Delphi llevado a cabo, incluyendo en él las tablas correspondientes a la última ronda del proceso y la interpretación de las mismas por parte del grupo coordinador. Finalmente, el artículo se cierra con la enumeración de algunas de las conclusiones a las que se ha llegado acerca del objeto de estudio.

\section{Aclaraciones metodológicas}

\subsection{El método Delphi}

Como ya sabemos, el método Delphi es una técnica de previsión subjetiva nacida en la década de los cuarenta y cuyo uso se extendió por los ámbitos empresariales y académicos especialmente desde los años setenta, sobre todo debido a las limitaciones de otras técnicas de previsión para obtener informaciones útiles ante situaciones de incertidumbre, habituales en nuestras sociedades. De acuerdo con Harold A. Listone y Murray Turoff (Landeta, 2002: 32), se puede definir como un "método de estructuración de un proceso de comunicación grupal que es efectivo a la hora de permitir a un grupo de individuos, como un todo, tratar un problema complejo".

Consiste en un proceso iterativo y sistemático en el que un conjunto de expertos es interrogado en rondas sucesivas a través de cuestionarios elaborados por los investigadores con el objetivo de obtener un consenso 
de opinión sobre el objeto de estudio o, al menos, una opinión grupal fidedigna, dado que en ocasiones la complejidad de los temas abordados no permite llegar al consenso como resultado de todo ese proceso.

Otras características sobre las que se fundamenta este método de investigación son, por una parte, la confidencialidad en las respuestas o el anonimato entre los participantes; gracias a esto último se evitan influencias negativas de los miembros dominantes, que a menudo pueden traducirse en la inhibición de algunos de los expertos, tal y como a veces sucede en los encuentros cara a cara (por ejemplo, en los grupos de discusión, que también pueden ser otra técnica de previsión subjetiva). Por otra parte, el tratamiento estadístico de las respuestas de los expertos, que permite que todas las opiniones individuales se tengan en cuenta a la hora de conformar una opinión grupal. Y finalmente, el feedback o retroalimentación controlado por los investigadores para evitar ruidos, es decir, información errónea, no entendible, no relevante, etc.; de hecho, en cada ronda los expertos deben conocer los resultados a los que se ha llegado en la ronda previa u otras informaciones que los investigadores consideren de interés. Por esta razón, Juan Antonio Gaitán Moya y José Luis Piñuel Raigada (1998: 137), entienden el método Delphi como "un procedimiento discursivo de carácter dialéctico, si bien mediado por los aplicadores y abierto a las aportaciones sucesivas del conjunto de los participantes".

\subsection{Justificación de la técnica elegida}

La utilización del Delphi como método de investigación científica en este trabajo ha estado motivada por varias razones. En primer lugar, se ha considerado adecuado para el objeto de estudio propuesto dado que, como se ha señalado, el objetivo de la presente investigación no era sólo realizar un análisis descriptivo del panorama de las RPEPE, sino también detectar tendencias de futuro para este sector; pues bien, el método Delphi se presenta como un instrumento de previsión y, por tanto, su fin es coherente con los objetivos propios de esta investigación.

En segundo lugar, del conjunto de técnicas científicas de previsión se ha optado por ésta debido a la ventaja derivada del feedback sobre el que se sustenta. Frente a las técnicas subjetivas de previsión de naturaleza individual, en las que la información procede de una sola persona o de un grupo sin que haya interacción entre sus miembros (por ejemplo, en las encuestas), en el método Delphi, como técnica grupal, los resultados son fruto de la interacción de un grupo de personas. Este aspecto es especialmente relevante, pues se parte de que esa interacción permite que el resultado final del proceso sea superior al que se conseguiría en el caso de utilizar técnicas de previsión individuales; o, lo que es lo mismo, que el resultado grupal sea superior a la suma de las aportaciones individuales de los participantes en el proceso. 
En tercer lugar, no podemos olvidar que tradicionalmente se ha establecido una línea divisoria entre los dos sectores de la actividad científica, el académico y el comercial; según Roger D. Wimmer y Joseph R. Dominick (1996: 13-14), en el primero las investigaciones tienen "un enfoque teorético o profesoral", mientras que los estudios del sector privado o comercial suelen ser "investigación aplicada, lo que significa que los resultados tienen por objeto servir de base para la toma de decisiones". Con la utilización del método Delphi en el presente trabajo se han intentado unificar ambos objetivos, en el sentido de aportar un mejor conocimiento teórico del mercado de las RPEPE y, al mismo tiempo, ser una herramienta útil para ayudar a la toma de decisiones por parte de los agentes implicados en el sector.

Para terminar, a todo ello se añade una última ventaja del Delphi como método de investigación científica. Nos referimos a la relación entre el bajo coste económico del proceso y el importante rendimiento en cuanto a la profundización en el objeto de estudio y a la obtención de datos relevantes. Es una ventaja meramente práctica, pero que también ha de ser reconocida como un factor determinante a la hora de elegir la técnica de investigación de este trabajo, dados los condicionantes materiales y económicos de partida.

\subsection{Método Delphi utilizado}

Para aclarar qué tipo de método Delphi ha sido utilizado seguiremos la clasificación recogida por Jon Landeta (2002: 130-143). Así, en cuanto al soporte se ha elegido el Delphi convencional, dadas las exigencias técnicas que planteaba la denominada conferencia Delphi, además de por otros de sus tradicionales inconvenientes. No obstante, el soporte ha sido un cuestionario en formato digital remitido a los expertos vía mail, de manera que en cierto modo también se podría hablar de un sistema mixto, en el que se han reducido los tiempos de recepción del feedback gracias a la utilización del correo electrónico.

Respecto a los objetivos perseguidos, se ha optado por un tipo de estudio orientado hacia el consenso, ya que se consideró que se partía de una posición consensual previa respecto a la naturaleza del problema analizado. El escenario de las revistas profesionales especializadas en publicidad en nuestro país es bastante estable, y por ello se han seguido los protocolos de la concepción original del Delphi con el fin de obtener una opinión grupal fidedigna respecto a su realidad y tendencias; eso sí, partiendo, en todo caso, de que esa orientación al consenso no significa que éste tuviera que conseguirse, principalmente por los condicionantes temporales que más adelante comentaremos cuando nos refiramos al criterio de parada.

En lo tocante a la utilidad o contribución esperada de la investigación, no se puede asignar este ejercicio Delphi a ninguna de las categorías descritas por Landeta ya que, como él mismo indica, se trata de estructuras ideales y el tipo de cada estudio viene definido por sus particularidades. Empero, sí podemos afirmar que 
este trabajo se ha planteado, de un lado, como una herramienta de análisis y comprensión de la realidad objeto de estudio; dicha realidad está suficientemente estructurada, pero se persiguió detectar la opinión grupal de los expertos respecto a sus carencias y oportunidades, de ahí que gran parte de las preguntas formuladas en el cuestionario soliciten en esta línea una respuesta descriptiva. Y, de otro lado, como un instrumento de información consensuada sobre las tendencias o posibles escenarios de futuro de la realidad objeto de estudio; en este objetivo se encuadran las opiniones de los expertos de carácter prospectivo. Finalmente, es importante señalar que el presente trabajo no ha sido diseñado pretendiendo ser un instrumento de decisión y creación de realidad, pues lo que se perseguía era adquirir conocimiento de la realidad, presente y futura, sin intentar modificarla; obviamente, ello no impide, como se indicó en párrafos anteriores, que junto a la utilidad científica de este estudio los propios expertos participantes u otros profesionales del sector publicitario puedan encontrar en él datos útiles para sus tomas de decisiones, lo cual sin duda supondría un valor añadido para los objetivos y planteamiento estratégico de la investigación realizada.

\section{Diseño de la investigación}

El proceso Delphi propuesto se ha desarrollado a los tres niveles discursivos a los que se refieren Gaitán Moya y Piñuel Raigada (1998: 138-139): el distribucional, relativo a la construcción de cada uno de los discursos particulares por parte de los expertos participantes, en nuestro caso a través de las respuestas a los cuestionarios aplicados; el relacional, referido a la comparación y acoplamiento que cada experto hace de su discurso con respecto al del resto de participantes en el proceso; y el nivel integrativo, conformado por la aportación de los investigadores responsables del estudio como síntesis de los distintos discursos particulares.

Para llevar a cabo la investigación se constituyó un grupo coordinador que, por razones operativas, se decidió que fuese reducido, pero que estuviese dentro de los márgenes que se recomiendan en los procesos Delphi (esto es, entre las dos y las cinco personas). El grupo estuvo integrado por los dos profesores universitarios que firman este artículo, especialistas en comunicación publicitaria, en uno de los casos, y en el estudio de productos periodísticos y editoriales, en el otro; se entendió que estas dos dimensiones eran complementarias para tratar el objeto de estudio seleccionado. Este grupo coordinador fue el encargado de aprobar el protocolo de trabajo y supervisar el desarrollo de la investigación, elaborar la lista de expertos participantes, diseñar los cuestionarios, analizar las respuestas, controlar el feedback e interpretar los resultados. 


\subsection{Selección de expertos}

Una vez decididos los medios técnicos a utilizar y consensuado el calendario de trabajo previsto, el grupo coordinador determinó los expertos participantes en el proceso en virtud de los criterios de selección previamente acordados; principalmente, el nivel de conocimientos, la capacidad predictiva y el grado de motivación. Se partió de la consideración de experto como aquella persona que por su situación o recursos personales puede aportar información, objetiva o subjetiva, útil para el logro de los fines planteados en la investigación, es decir, para conocer la realidad de las RPEPE y plantear tendencias o escenarios de futuro al respecto. En este sentido se prefirió seleccionar participantes que fuesen especialistas en el tema objeto de estudio y que, al mismo tiempo, estuviesen implicados activamente en esa realidad.

De todas las posibilidades planteadas, se optó por tomar como expertos participantes a los directivos de las principales revistas del sector en nuestro país. El grupo coordinador fue consciente de la existencia de otros posibles expertos (teóricos de la comunicación y la publicidad, empresarios del mercado editorial, directivos de agencias publicitarias, responsables de comunicación y marketing de las empresas anunciantes, etc.); sin embargo, se eligió el grupo señalado al tratarse esta investigación de un primer paso en el conocimiento y prospección respecto al objeto de estudio, pudiéndose completar en sucesivas fases con las opiniones de esos y otros grupos de especialistas en función de disponibilidades presupuestarias y técnicas.

Los expertos que confirmaron su participación inicial en el estudio fueron, por orden alfabético, los que se detallan a continuación, acompañados del cargo que ostentaban en el momento de la realización del mismo (en el segundo semestre del año 2007):

- Daniel Campo, director de la revista El Publicista de la publicidad, la comunicación y el marketing.

- Gabriel González-Andrío, director de la revista El Periódico de la Publicidad.

- Manuel G. Carbajo, director de la revista Ipmark. Información de publicidad y marketing.

- $\quad$ Manuel de Luque, director de la revista Anuncios. Semanario de publicidad y marketing.

- $\quad$ Paloma Bas, directora de la revista Interactiva. Revista de la comunicación y el marketing digital.

- $\quad$ Penélope García, directora de la revista Estrategias de comunicación y marketing.

- $\quad$ Poli Sánchez, directora de la revista MK. Marketing + Ventas.

- $\quad$ Raúl Sánchez, redactor jefe de la revista Control. La primera revista de publicidad.

$64\left|n^{\circ} 7\right|$ doxa.comunicación 
Concluyeron el proceso Delphi todos ellos, excepto el representante de El Periódico de la Publicidad. Los abandonos, que pueden distorsionar seriamente el resultado final del proceso, suelen oscilar en los trabajos publicados entre un 20 y un 30\% según Michal J. Bardecki (Landeta, 2002: 122), por lo que el índice de abandono en el presente estudio ha sido bajo: no llega al 13\%. Teniendo esto en cuenta, el número final de expertos participantes ha sido de 7, científicamente válido por dos razones. Primero, porque es el umbral mínimo orientativo que en los inicios de la técnica los investigadores de la Rand Corporation de EE.UU. establecían para garantizar la precisión de la estimación grupal. Y segundo, porque, además, partiendo de lo restringido del colectivo de expertos que el grupo coordinador consideró que tenía que participar en el estudio, se puede afirmar que el número de participantes en la investigación es relativamente elevado, al estar representadas en el panel de expertos las principales revistas del sector en nuestro país.

\subsection{Rondas y cuestionarios}

Una vez seleccionados los expertos, se les mandó vía mail un cuestionario sobre el objeto de estudio formado por 14 preguntas dividas en dos bloques. El primero, de 6 preguntas, estaba constituido, por una parte, por 5 cuestiones relativas a la publicación que dirige cada uno de los expertos participantes; se les requería información sobre el segmento de mercado al que se dirige su revista, su posicionamiento y ventaja diferencial, la evolución de la difusión, las fuentes de financiación o los principales retos empresariales a corto, medio y largo plazo. Las respuestas a estas 5 preguntas no fueron utilizadas en el proceso Delphi, sino que con ellas se perseguía únicamente recabar información teórica respecto al objeto de estudio para contrastarla con la que ya tenían los miembros del grupo coordinador. Por tal razón y para garantizar la fiabilidad de los datos aportados por los panelistas, se garantizó la confidencialidad de las respuestas de esta parte del cuestionario, comprometiéndose los investigadores a no utilizarlas en fases posteriores del proceso iterativo. Por otra parte, en este bloque también se incluyó otra cuestión que tampoco formó parte del proceso Delphi y que se refería a la opinión de los expertos sobre las que consideraban revistas del sector con mayor impacto en nuestro país; esta pregunta era importante formularla no sólo por el interés intrínseco de las respuestas, sino también para certificar que los expertos participantes en el proceso representaban a las cabeceras que los propios profesionales calificaban como de mayor impacto en el sector.

Las restantes 8 preguntas, pertenecientes al segundo bloque del documento enviado a los expertos, son las que fueron utilizadas en el proceso Delphi. En el cuestionario de esta primera ronda se plantearon preguntas abiertas para, a partir de las contestaciones obtenidas, poder extraer los ítems que luego se incorporasen como posibilidades de respuesta en el cuestionario de la siguiente ronda del proceso, garantizando así que la influencia del grupo coordinador en el diseño de ese segundo cuestionario fuese mínima. 
Para posibilitar esto, las preguntas fueron formuladas con el fin de facilitar el procesamiento posterior de las respuestas por los investigadores; en este sentido, para evitar respuestas que fuesen difíciles de convertir en ítems de cara al segundo cuestionario, en las cuestiones planteadas se solicitaba de los expertos que enumerasen aspectos como las funciones o necesidades que deben satisfacer las RPEPE, los factores internos y externos que condicionan el mercado de estas publicaciones, fortalezas y debilidades del mismo, competidores actuales, principales tendencias o funciones que desempeñan las páginas web de esas cabeceras, entre otros.

Tal y como acabamos de avanzar, sobre la base de las respuestas dadas a las preguntas abiertas de esa primera fase se diseñó el cuestionario que se utilizó en la segunda ronda. En ese diseño se tuvo en cuenta que fueran cuestiones acordes con el tipo de metodología Delphi utilizada, esto es, que posteriormente pudieran ser sometidas a tratamiento estadístico para extraer una respuesta estadística de grupo. El resultado fueron, por una parte, 7 preguntas cerradas en las que, a partir de una serie de ítems -ordenados alfabéticamente para evitar condicionamientos en las respuestas- y en función de un determinado criterio que se indicaba en el enunciando de cada pregunta, se solicitaban valuaciones por parte de los expertos de acuerdo con una escala previamente definida, comprendida siempre entre los valores 0 y 5 ; con ello se permitía fácilmente la integración numérica de las respuestas. Se prefirió la valuación frente a la jerarquización de los ítems debido a que en la mayor parte de las cuestiones el número de opciones de respuesta era bastante elevado, con lo que era más sencillo para los expertos responder por valuación que por jerarquización. Por otra parte, en la pregunta 8 se solicitaba de los panelistas una respuesta única de entre las posibilidades propuestas a partir de los datos recabados en esa misma pregunta del primer cuestionario.

\subsection{Feedback y criterio de parada}

En esta segunda ronda el feedback se limitó únicamente a la información facilitada por los expertos en la primera fase tras ser procesada por el equipo coordinador. Fue labor de los investigadores interpretar esos datos de forma lo más objetiva posible seleccionando las opiniones más significativas para incorporarlas al proceso iterativo posterior y manteniendo en todo momento el sentido de las respuestas y sin perder información real. En el diseño previo de la investigación ya se había decidido no facilitar información estadística en esta segunda ronda debido a que, como a veces reconocen los especialistas en metodología Delphi, es discutible que realmente esta información enriquezca el proceso. Esto, por supuesto, teniendo en cuenta lo que señala Juan Báez y Pérez de Tudela (2007: 205), referente a que "la mayor amenaza para el análisis es que se reduzca a datos numéricos toda la información aportada por los expertos y, con ello, infrautilizar la riqueza de significados de los datos cualitativos". 
Con esta segunda fase se puso fin al proceso iterativo, dado que en el diseño previo del estudio ya se había establecido de antemano el criterio de parada, en función del calendario de trabajo acordado y para evitar la desmotivación entre los expertos participantes. Tenemos que tener en cuenta que aunque no se pueda alcanzar totalmente el consenso o la estabilidad en la respuestas, lo habitual en los estudios Delphi es limitarse a dos o tres rondas; además, según afirma Landeta (2002: 101-102), "conversaciones mantenidas con empresas especializadas en el empleo de esta técnica para atender comercialmente a las solicitudes prospectivas de sus clientes nos han confirmado que estas limitaciones temporales, presupuestarias y la dificultad de mantener el compromiso de los expertos hacen que rara vez se llegue a la tercera ronda".

Para presentar los resultados del proceso se procedió a la integración de las respuestas recibidas en una estimación grupal, valorando por igual esas respuestas individuales independientemente del experto al que perteneciesen. Al ser valuaciones la mayor parte de las preguntas de la segunda ronda, lo que se hizo fue calcular una medida de tendencia central de los valores de cada ítem en cada una de las preguntas y se reordenaron esos ítems de acuerdo con esos valores centrales obtenidos. La medida de tendencia central utilizada fue la mediana y no la media porque con esta última se daría excesivo peso a las respuestas con valores extremos, corriéndose el riesgo de que se desvirtuase la opinión grupal.

\section{Principales resultados}

Una vez aclarado el objeto de estudio y los objetivos de la presente investigación, explicado el diseño metodológico y detallados los diferentes pasos en el proceso de ejecución del mismo, pasamos a presentar los resultados más relevantes. Para una mayor claridad en la exposición y teniendo en cuenta que entre la primera y segunda ronda no se ha llevado a cabo una explotación estadística de las respuestas, se exponen principalmente los resultados finales del proceso iterativo, recogiéndose en menor medida la evolución de las opiniones a través de dicho proceso.

En las tablas adjuntas se han ordenado los distintos ítems de respuesta de mayor a menor consenso. Por consiguiente, los primeros puestos son ocupados por aquellos ítems en los que el valor de la mediana es superior, y cuando la mediana correspondiente a varios ítems tiene el mismo valor entonces han sido ordenados de menor a mayor desviación típica. El objetivo ha sido poder visualizar fácilmente a través de las tablas el grado de consenso o disenso de forma gradual. Todo ello se complementa con la información precisa para interpretar correctamente los resultados.

Comenzando con un análisis descriptivo de la situación actual de las RPEPE, se pidió a los expertos participantes su opinión acerca de los factores, internos y externos al negocio editorial, que en la actualidad 
condicionan el mercado de estas revistas. Tras proceder a la integración de las respuestas individuales obtenidas en la primera ronda del proceso Delphi, en la segunda fase se les solicitó que valorasen cada uno de los ítems obtenidos en una escala de 0 a 5 , puntuando con 0 aquellos factores que consideraban que no condicionan en nada el mercado editorial y con 5 los que pensaban que lo condicionan mucho.

Pues bien, el principal factor condicionante para los expertos, y en el que hay un mayor consenso de todos los ítems propuestos, es el referido a la dependencia de los ingresos por publicidad (véase tabla I). En un segundo escalón, pero con un grado de disenso algo mayor, los panelistas creen que condicionan el mercado de las RPEPE la dependencia de ingresos por suscripción, las reducidas plantillas con las que cuentan, el solapamiento entre las distintas cabeceras y otras cuestiones obvias como las necesidades informativas de los profesionales de la publicidad o la propia situación económica del sector y del país en general.

Tabla I. Factores internos y externos al negocio editorial que condicionan en la actualidad el mercado de las RPEPE

\begin{tabular}{|l|c|c|c|c|}
\hline Puesto & Ítem & Mediana & Media & Desv. \\
\hline 1 & Dependencia de los ingresos por publicidad & 5 & 4,57 & 0,73 \\
\hline 2 & Dependencia de los ingresos por suscripción & 4 & 3,71 & 1,03 \\
\hline 3 & Reducidas plantillas para satisfacer las demandas \\
y necesidades de los lectores & 4 & 3,71 & 1,03 \\
\hline 4 & Solapamiento entre las distintas publicaciones & 4 & 3,57 & 1,05 \\
\hline 5 & $\begin{array}{c}\text { Necesidades informativas de los profesionales del } \\
\text { sector, que demandan conocer los cambios } \\
\text { que se están produciendo }\end{array}$ & 4 & 3,71 & 1,28 \\
\hline 6 & $\begin{array}{c}\text { Situación económica del sector y del país en general } \\
\text { y con escasez de compradores }\end{array}$ & 4 & 2,71 & 1,58 \\
\hline 7 & $\begin{array}{c}\text { Exceso de cabeceras para un mercado reducido } \\
\text { las necesidades y demandas de los lectores }\end{array}$ & 3 & 3,57 & 0,73 \\
\hline 9 & $\begin{array}{c}\text { Poca transparencia de los subsectores implicados, } \\
\text { principalmente de los anunciantes, que no facilitan } \\
\text { datos o dificultan su publicación }\end{array}$ & 3 & 2,86 & 1,12 \\
\hline
\end{tabular}

$68\left|n^{\circ} 7\right|$ doxa.comunicación 
Antonio J. Baladrón Pazos y Beatriz Correyero Ruiz

\begin{tabular}{|l|c|c|c|c|}
\hline 10 & Procesos de concentración y fusión de los grupos editoriales & 3 & 2,43 & 1,29 \\
\hline 11 & $\begin{array}{c}\text { Altos costes de distribución, que condicionan los envíos } \\
\text { de las revistas a España y otros países }\end{array}$ & 3 & 3,29 & 1,58 \\
\hline 12 & $\begin{array}{c}\text { Altos costes fijos, que provocan elevado precio } \\
\text { del ejemplar y de las suscripciones }\end{array}$ & 3 & 3,43 & 1,68 \\
\hline 13 & $\begin{array}{c}\text { Escasez de medios para satisfacer las necesidades } \\
\text { y demandas de los lectores }\end{array}$ & 1 & 1,57 & 1,59 \\
\hline
\end{tabular}

Fuente: Elaboración propia

Asimismo, otros factores algo menos importantes son, por este orden, los siguientes: el exceso de cabeceras para un mercado reducido y con escasez de compradores; la competencia actual del medio Internet para satisfacer las mismas necesidades informativas; la poca transparencia de los subsectores implicados, especialmente de los anunciantes, que no facilitan datos suficientes o dificultan su publicación; los procesos de concentración y fusión de los grupos editoriales responsables de estas cabeceras; los altos costes de distribución de las revistas, tanto dentro de España como hacia el extranjero; y los altos costes fijos, que provocan que tanto las suscripciones como los precios por ejemplar sean ciertamente elevados. Finalmente, los expertos opinan que, salvo lo indicado en lo concerniente a las plantillas con las que cuentan, estas cabeceras disponen de medios suficientes para satisfacer las necesidades y demandas de los lectores; no en vano este ítem de respuesta ha sido el menos valorado como factor condicionante del mercado de las RPEPE, si bien, como haremos cuando se interprete la tabla III, esto puede ser cuestionado.

En la formulación de esta pregunta no se detallaba si esos factores condicionaban positiva o negativamente el mercado de las revistas especializadas en publicidad. En consecuencia, es importante atender a otras dos preguntas que se formularon sobre las principales fortalezas y debilidades de las RPEPE, con el fin de profundizar más en la situación actual de estas cabeceras.

Si empezamos por las fortalezas (véase tabla II) podemos dejar patente que existe un acentuado grado de consenso a la hora de seleccionar los puntos fuertes más importantes de las RPEPE. El primero de ellos, a juicio de los expertos, es el amplio conocimiento que tienen del mundo publicitario, de sus necesidades y expectativas. El segundo es el arraigo o tradición con el que en la actualidad estas revistas cuentan en el sector. Y el tercero, la buena cobertura informativa que ofrecen a los lectores de todo lo que acontece en el ámbito publicitario. 
Tabla II. Principales fortalezas de las RPEPE

\begin{tabular}{|l|c|c|c|c|}
\hline Puesto & Ítem & Mediana & Media & Desv. \\
\hline 1 & Amplio conocimiento del sector, de sus necesidades & 5 & 4,71 & 0,45 \\
y expectativas & Arraigo en el sector & 5 & 4,57 & 0,49 \\
\hline 3 & Buena cobertura informativa de todo lo que acontece \\
en el sector & 4 & 4,29 & 0,7 \\
\hline 4 & Buena imagen entre los profesionales del sector & 4 & 3,71 & 1,03 \\
\hline 5 & Buenos y veteranos equipos de redacción y firmas y & 4 & 3,71 & 1,03 \\
\hline 6 & colaboradores de prestigio & 4 & 3,43 & 1,18 \\
\hline 7 & Buenos equipos de ventas & 4 & 3,57 & 1,59 \\
\hline 8 & Profundidad en el tratamiento de los contenidos & 4 & 3,86 & 1,64 \\
\hline 9 & Cación fluida y constante con las agencias y los medios & 4 & 3,71 & 1,67 \\
\hline 10 & Calidad de los contenidos, rigor y profesionalidad & 4 & 3,57 & 1,68 \\
\hline 11 & en el tratamiento de los temas & 3 & 2,71 & 1,58 \\
\hline 12 & Periodicidad adecuada & 3 & 2,43 & 1,59 \\
\hline 13 & Flexibilidad para adaptarse a las demandas del mercado & 3 & 2,86 & 1,73 \\
\hline 14 & Reducido número de publicaciones & 2 & 1,57 & 1,18 \\
\hline
\end{tabular}

Fuente: Elaboración propia

Otras fortalezas destacadas por los expertos participantes en el proceso Delphi tienen que ver tanto con la buena imagen de estas cabeceras entre los profesionales como con los equipos humanos encargados de su realización; en este sentido, valoran de manera importante los buenos y veteranos equipos de redacción, el hecho de contar con firmas y colaboradores de prestigio y los buenos equipos de ventas. Le siguen, con un grado de disenso superior, diversos aspectos relacionados con la calidad, profundidad o rigor en los contenidos, la buena calidad de impresión o, también como una fortaleza destacada, la relación fluida y constante que mantienen con medios y agencias.

$70 \mid n^{\circ} 7$ | doxa.comunicación 
Por último, similar grado de disenso al de estos últimos puntos se aprecia en la inferior valoración otorgada por los expertos participantes a ítems como la adecuada periodicidad de estas cabeceras, el buen precio del ejemplar y las suscripciones ${ }^{1}$ y la flexibilidad para adaptarse a las demandas del mercado.

En cuanto a las debilidades (véase tabla III), las valoraciones otorgadas por los expertos a los ítems resultado de la integración de las respuestas individuales de la primera ronda son globalmente inferiores. Una tercera ronda, completando los ítems de respuesta con aportaciones por parte del grupo coordinador, posiblemente hubiera enriquecido el proceso, pero no se llevó a cabo por incoherencia con los planteamientos metodológicos iniciales y por el criterio de parada consensuado por el grupo coordinador desde un primer momento.

Tabla III. Principales debilidades de las RPEPE

\begin{tabular}{|l|c|c|c|c|}
\hline Puesto & Ítem & Mediana & Media & Desv. \\
\hline 1 & Falta de columnistas u opiniones internacionales & 4 & 3,86 & 0,64 \\
\hline 2 & $\begin{array}{c}\text { Abuso en la realización de especiales con el fin de } \\
\text { obtener ingresos publicitarios }\end{array}$ & 4 & 0,93 \\
\hline 3 & $\begin{array}{c}\text { Poca visibilidad, al venderse mediante suscripción } \\
\text { o a través de un circuito limitado de puntos de venta }\end{array}$ & 4 & 3,14 & 1,36 \\
\hline 4 & $\begin{array}{c}\text { Pocas suscripciones por empresas, por lo que sólo llegan } \\
\text { a directivos y no al resto de profesionales }\end{array}$ & 4 & 3 & 1,41 \\
\hline 5 & Solapamiento de los temas tratados entre las distintas cabeceras & 3 & 3,14 & 0,83 \\
\hline 6 & Escasa visión y tratamiento de la revolución digital & 3,14 & 0,83 \\
\hline 7 & $\begin{array}{c}\text { Escasez de recursos para satisfacer las demandas } \\
\text { y necesidades de los lectores }\end{array}$ & 3 & 3,14 & 0,83 \\
\hline 8 & $\begin{array}{c}\text { Excesiva dependencia de la publicidad, con la } \\
\text { consiguiente pérdida de independencia }\end{array}$ & 3 & 3,29 & 1,03 \\
\hline 9 & $\begin{array}{c}\text { Reducidas plantillas para satisfacer las } \\
\text { necesidades y demandas de los lectores }\end{array}$ & 4 & 4 \\
\hline
\end{tabular}

1 Recordemos, en todo caso, que en las repuestas a la pregunta mencionada anteriormente, recogidas en la tabla I, los panelistas dejaban entrever que esos precios los consideraban elevados, motivados por los altos costes fijos de las cabeceras, lo que justifica esta inferior valoración, aun cuando los siguen considerando fortaleza. 
Las revistas profesionales especializadas en publicidad en España: resultados de un estudio Delphi

\begin{tabular}{|l|c|c|c|c|}
\hline 10 & Mercado reducido e inconsistente & 3 & 2,43 & 1,05 \\
\hline 11 & Escasa diferenciación entre cabeceras & 3 & 2,57 & 1,4 \\
\hline 12 & $\begin{array}{c}\text { Superficialidad o falta de profundización } \\
\text { en el tratamiento informativo de los temas }\end{array}$ & 2 & 2,14 & 0,83 \\
\hline 13 & Elevados precios & 2 & 2,29 & 1,03 \\
\hline 14 & Poca tirada & 2 & 2,14 & 1,64 \\
\hline 15 & Reducido tamaño & 1 & 0,71 & 0,7 \\
\hline 16 & Poca consistencia empresarial en algunos casos & 1 & 1,71 & 1,28 \\
\hline 17 & Muy especializadas & 0 & 0,43 & 0,73 \\
\hline 18 & Muy técnicas & 0 & 0,43 & 0,73 \\
\hline
\end{tabular}

Fuente: Elaboración propia

Teniendo esto en cuenta, podemos afirmar que para los expertos las debilidades más importantes de las RPEPE son, por este orden: la falta de columnistas o firmas internacionales; el abuso en la realización de especiales con el fin de obtener ingresos publicitarios; la poca visibilidad que tienen en la sociedad, al venderse mediante suscripción o a través de un circuito limitado de puntos de venta; relacionado con esto último, también la poca visibilidad en las empresas del sector, debido a que hay pocas suscripciones por cada empresa, lo que provoca que habitualmente lleguen sólo a directivos y no al resto de profesionales; y el excesivo solapamiento de temas entre las distintas cabeceras existentes en el mercado.

Otras debilidades señaladas como algo menos importantes con un cierto grado de consenso son la escasa visión y tratamiento de estas cabeceras en lo que se refiere a la revolución digital, la escasez de recursos para satisfacer las demandas y necesidades de los lectores, la excesiva dependencia de la publicidad, las reducidas plantillas, el hecho de tratarse de un mercado reducido e inconsistente o la escasa diferenciación entre cabeceras.

En algunas de estas opiniones grupales se podrían apreciar ciertas contradicciones con respecto a las manifestadas en otras respuestas. En lo referente a la excesiva dependencia de la publicidad, aunque según el juicio subjetivo de los panelistas está en el puesto octavo de debilidades, cuando los expertos se referían a los principales factores condicionantes del mercado de las RPEPE lo consideraban el principal factor condicionante (recuérdese lo expuesto en la tabla I). Podríamos considerar que se incurre en contradicciones, pero eso debe ser matizado, por dos razones. En primer lugar, porque la dependencia de la publicidad puede 
ser entendida como un condicionante claro del mercado, pero no necesariamente como un aspecto negativo a destacar como propio de estas cabeceras, tal y como constatan los expertos. Y, en segundo lugar, porque la segunda principal debilidad que consideran los directivos participantes está también estrechamente ligada a ésta; nos referimos al abuso en la realización de especiales con el fin de obtener ingresos publicitarios. Por tanto, globalmente podemos concluir que los expertos sí creen que la dependencia de la publicidad sea uno de los principales condicionantes y en ocasiones debilidad de estas cabeceras.

Por su parte, las reducidas plantillas ya las subrayaban los expertos como uno de los más importantes factores condicionantes (tabla I), por lo que las respuestas aquí dadas redundan en esa misma idea, si bien defienden la existencia de debilidades más relevantes. Sin embargo, sí pudiera existir contradicción entre lo recogido en la tabla I y lo que aquí manifiestan los expertos respecto a si estas cabeceras tienen recursos escasos para satisfacer las demandas y necesidades de los lectores; así, si los expertos no consideraban prácticamente ese ítem como un factor condicionante, en esta ocasión sí creen que se trata de una debilidad digna de ser mencionada. Queremos dejar manifiesta esta posible contradicción, concluyendo, de cualquier modo, que para los expertos sí se podría tratar de una importante debilidad de estas revistas dado que, una vez más, el término condicionante es más genérico y amplio que el de debilidad.

El resto de los ítems recogidos en las respuestas tras la integración de las estimaciones individuales de la primera ronda no son debilidades relevantes para los expertos; de hecho, según la opinión grupal no lo son la superficialidad o falta de profundización en los temas (en coherencia con las respuestas a la pregunta recogida en la tabla II, en donde se reflejaba como fortaleza), los elevados precios (igualmente en conexión con lo recogido en la tabla II, en la que el precio también era un aspecto reseñado como relativamente positivo), la poca tirada de esas cabeceras o su reducido tamaño, entre otras cuestiones.

En otro orden de cosas, fueron también muy bajas las valoraciones dadas por los expertos a los ítems de la pregunta relativa a los principales competidores de las RPEPE en la actualidad (véase tabla IV).

Tabla IV. Actuales competidores de las RPEPE

\begin{tabular}{|l|c|c|c|c|}
\hline Puesto & Ítem & Mediana & Media & Desv. \\
\hline 1 & Portales y websites sobre publicidad & 3 & 2,43 & 1,18 \\
\hline 2 & Publicaciones económicas & 2 & 1,86 & 0,99 \\
\hline 3 & Foros en Internet sobre publicidad & 2 & 1,71 & 1,03 \\
\hline 4 & Otras publicaciones de comunicación o marketing & 2 & 2,29 & 1,48 \\
\hline
\end{tabular}


Las revistas profesionales especializadas en publicidad en España: resultados de un estudio Delphi

\begin{tabular}{|l|c|c|c|c|}
\hline 5 & Revistas extranjeras sobre publicidad & 2 & 2,43 & 1,5 \\
\hline 6 & Blogs sobre publicidad & 2 & 2,29 & 1,83 \\
\hline 7 & Publicaciones generalistas & 1 & 1,14 & 1,12 \\
\hline
\end{tabular}

Fuente: Elaboración propia

De manera general podemos decir que los portales y los websites sobre publicidad son considerados los únicos competidores (en la tabla II, con un índice de valoración similar, la competencia del medio Internet ya se señalaba como factor condicionante del mercado de las RPEPE), aunque siguen sin ser valorados como competidores verdaderamente importantes y el grado de consenso al respecto no es destacable. Los expertos dieron poca importancia como rivales en el mercado a otros agentes como las publicaciones económicas, los foros en Internet sobre publicidad, otras publicaciones de comunicación o marketing y las revistas extranjeras especializadas en publicidad. Finalmente, tampoco se consideró como competidor a las publicaciones generalistas.

Por todo ello, de las respuestas de los panelistas en las dos rondas se podría deducir que no existen en el mercado competidores realmente relevantes a la hora de satisfacer las necesidades a las que dan respuesta las RPEPE; ahora bien, el primer puesto otorgado a portales y websites por parte de los expertos permite vislumbrar la importancia que puede estar cobrando Internet, como también se constatará más adelante en los datos recogidos en la tabla VI.

Ante lo complejo de esta situación, también se consultó a los expertos respecto a la utilización de Internet que hacen las RPEPE. En concreto, en el segundo cuestionario se les pidió que valorasen en qué medida están desarrolladas determinadas funciones que desempeñan las webs de estas revistas y que previamente habían sido apuntadas por los ellos mismos en la primera ronda (véase tabla V).

Tabla V. Funciones que desempeñan las páginas web de las RPEPE

\begin{tabular}{|l|c|c|c|c|}
\hline Puesto & Ítem & Mediana & Media & Desv. \\
\hline 1 & $\begin{array}{c}\text { Transmitir con mayor inmediatez que la revista } \\
\text { en papel las últimas noticias sobre el sector }\end{array}$ & 4 & 4,07 \\
\hline 2 & Actuar como hemeroteca de las informaciones publicadas & 4 & 3,57 & 1,18 \\
\hline 3 & $\begin{array}{c}\text { Utilizar la plataforma virtual como gancho } \\
\text { para atraer lectores a la revista en papel }\end{array}$ & 4 & 3,43 & 1,59 \\
\hline 4 & $\begin{array}{c}\text { Favorecer la venta } \text { on line de otros productos } \\
\text { de la editorial de la revista }\end{array}$ & 3 & 3,57 & 0,73 \\
\hline
\end{tabular}

$74\left|n^{\circ} 7\right|$ doxa.comunicación 
Antonio J. Baladrón Pazos y Beatriz Correyero Ruiz

\begin{tabular}{|l|c|c|c|c|}
\hline 5 & Generar debate sobre temas de actualidad para el sector & 3 & 2,57 & 0,9 \\
\hline 6 & $\begin{array}{c}\text { Complementar la versión en papel de la revista, } \\
\text { prestando servicios añadidos }\end{array}$ & 3 & 3,14 & 1,12 \\
\hline 7 & Adelantar al lector parte de los contenidos de las revista en papel & 3 & 3,43 & 1,18 \\
\hline 8 & $\begin{array}{c}\text { Tratar de modo más dinámico y audiovisual } \\
\text { los temas que afectan al sector }\end{array}$ & 3 & 2,43 & 1,29 \\
\hline 9 & Incluir publicidad para contribuir a la financiación de la revista & 3 & 2,86 & 1,46 \\
\hline 10 & Ser fuente de información sobre la revista en papel & 3 & 3,14 & 1,46 \\
\hline 11 & $\begin{array}{c}\text { Servir de punto de encuentro para la oferta } \\
\text { y demanda de servicios publicitarios }\end{array}$ & 2 & 2 & 0,93 \\
\hline 12 & $\begin{array}{c}\text { Presentarse como espacio virtual para } \\
\text { colgar informaciones publicadas en papel }\end{array}$ & 2 & 2,71 & 1,48 \\
\hline
\end{tabular}

Fuente: Elaboración propia

Las funciones más desarrolladas son, en primer lugar, las de transmitir con mayor inmediatez que la revista en papel las últimas noticias sobre el sector, actuar como hemeroteca de las informaciones publicadas y utilizar la plataforma virtual como gancho para atraer lectores a la revista en papel. Se trata de funciones que las versiones digitales de estas publicaciones comparten con las de otras publicaciones de distinta naturaleza, que en gran parte de los casos también utilizan la web con fines parecidos.

En segundo lugar, con grados de consenso variables algunas otras funciones menos desarrolladas son: favorecer la venta on line de otros productos de la editorial de la revista, generar debate sobre temas de actualidad para el sector, complementar la versión en papel prestando valores añadidos, adelantar al lector parte de los contenidos de la revista tradicional, tratar de modo más dinámico y audiovisual los temas que afectan al mundo publicitario, incluir publicidad para contribuir a la financiación del negocio o ser fuente de información sobre la cabecera en papel.

Como ya se señaló en los primeros párrafos de este trabajo, el objetivo de la investigación Delphi llevada a cabo era realizar un análisis descriptivo del panorama de las RPEPE, pero también detectar tendencias de futuro. Por eso, los expertos fueron preguntados no sólo por la situación actual de estas cabeceras, sino también por la situación deseable o por la evolución del mercado. 
Respecto a lo primero, se invitó a los participantes a que expresasen las funciones o necesidades que, a su juicio, deben satisfacer las RPEPE. Tras recabar sus opiniones, en la segunda ronda del estudio, al igual que hicieron en el resto de preguntas, valoraron cada uno de los ítems en una escala de 0 a 5 , siendo en este caso 0 nada importante y 5 muy importante. Los resultados finales (véase tabla VI) corroboran el alto grado de consenso que ya se había alcanzado en la ronda inicial del proceso.

Tabla VI. Funciones o necesidades que deben satisfacer las RPEPE

\begin{tabular}{|l|c|c|c|c|}
\hline Puesto & Ítem & Mediana & Media & Desv. \\
\hline 1 & $\begin{array}{c}\text { Interpretar y reflexionar sobre los cambios que se están } \\
\text { produciendo en el sector, profundizando en el análisis } \\
\text { del mercado y los temas de fondo }\end{array}$ & 5 & 0 \\
\hline 2 & $\begin{array}{c}\text { Informar a los profesionales sobre la actualidad } \\
\text { y tendencias del sector de la publicidad }\end{array}$ & 5 & 4,86 & 0,35 \\
\hline 3 & $\begin{array}{c}\text { Opinar y recoger opinión de los profesionales de la publicidad, } \\
\text { creando debate para enriquecer el sector }\end{array}$ & 5,86 & 0,35 \\
\hline 4 & $\begin{array}{c}\text { Ser herramientas útiles para el día a día de los profesionales } \\
\text { del sector, ayudándoles en la realización de campañas, } \\
\text { planificación de medios, etc. }\end{array}$ & 5 & 4,29 & 1,16 \\
\hline 5 & $\begin{array}{c}\text { Informar a los estudiantes y aspirantes a la profesión } \\
\text { sobre la actualidad y tendencias del sector de la publicidad }\end{array}$ & 4 & 4,14 & 0,64 \\
\hline 6 & $\begin{array}{c}\text { Informar a los profesionales sobre la actualidad y tendencias } \\
\text { externas al sector de la publicidad pero que } \\
\text { pueden afectarle indirectamente }\end{array}$ & 3 & 3,14 & 0,99 \\
\hline
\end{tabular}

Fuente: Elaboración propia

Existe unanimidad al defender que estas revistas tienen como misión interpretar y reflexionar sobre los cambios que se están produciendo en el sector, profundizando en el análisis del mercado y los temas de fondo; todos los expertos concedieron el valor máximo a este ítem. Junto a la interpretación y la profundización, en segundo lugar los panelistas consideraron fundamental la función de informar a los profesionales sobre la actualidad y tendencias del sector y la de opinar y recoger opinión de esos profesionales con el fin de generar debate. En consecuencia, las tres funciones clásicas de los productos periodísticos -información, interpretación y opinión- son valoradas prácticamente a un mismo

76 | n 7 | doxa.comunicación 
nivel de importancia, si bien prima en todo caso la interpretación y profundización como función esencial de las RPEPE.

Por otra parte, los expertos no olvidan que esas funciones clásicas del periodismo han de traducirse en convertir a estas cabeceras en herramientas útiles para el día a día de los profesionales del sector, ayudándoles en cuestiones como la realización de campañas, la planificación de medios, etc. Esta función pragmática es también muy valorada, como se puede apreciar en los datos recogidos en la tabla VI; no obstante, la desviación típica de los datos de este ítem revela un grado de consenso sensiblemente inferior.

En un quinto puesto, es primordial para los expertos que las RPEPE sean un instrumento de información al servicio no sólo de los profesionales, sino incluso de los estudiantes y aspirantes a la profesión. Y, por último, algo menos de consenso existe en cuanto a que tengan como misión informar a los profesionales del sector de la publicidad sobre la actualidad o tendencias externas al propio sector, aunque ellas puedan afectarles indirectamente.

En lo tocante a las tendencias para el futuro de las RPEPE (véase tabla VII), existe un significativo consenso respecto a que las más relevantes son las que tienen que ver con el mayor y mejor aprovechamiento de las nuevas tecnologías de la información y la comunicación. Así, se consideran tendencias más importantes la necesidad de potenciar la digitalización y el aprovechamiento de Internet para dar valor añadido al profesional y la de potenciar la interactividad con los lectores o potenciales lectores, junto a la de utilizar el website y el newsletter para enganchar al target e incitarle a la suscripción (tal y como también se deducía de las repuestas recogidas en la tabla $\mathrm{V}$ ).

Tabla VII. Tendencias para el futuro de las RPEPE

\begin{tabular}{|l|c|c|c|c|}
\hline Puesto & Ítem & Mediana & Media & Desv. \\
\hline 1 & $\begin{array}{c}\text { Potenciar la digitalización y el aprovechamiento de Internet } \\
\text { para dar valor añadido al profesional }\end{array}$ & 5 & 4,71 & 0,45 \\
\hline 2 & Potenciar la interactividad con los lectores o posibles lectores & 5 & 4,43 & 0,73 \\
\hline 3 & $\begin{array}{c}\text { Utilizar los website y newsletter para enganchar } \\
\text { al target } \text { e incitarle a la suscripción }\end{array}$ & 4 & 4,43 & 0,49 \\
\hline 4 & $\begin{array}{c}\text { Incrementar el rigor en el contenido y el análisis de } \\
\text { los temas, llevando a cabo una mayor } \\
\text { profundización en los temas tratados }\end{array}$ & 4 & 4,14 & 0,64 \\
\hline
\end{tabular}


Las revistas profesionales especializadas en publicidad en España: resultados de un estudio Delphi

\begin{tabular}{|l|c|c|c|c|}
\hline 5 & Acercarse en mayor grado al mundo universitario & 4 & 3,57 & 0,9 \\
\hline 6 & Propiciar una mayor participación de los profesionales del sector & 4 & 3,14 & 1,12 \\
\hline 7 & Tener un contacto más estrecho y constante con la audiencia & 4 & 3,57 & 1,18 \\
\hline 8 & $\begin{array}{c}\text { Potenciar el uso de nuevos formatos publicitarios en sus } \\
\text { páginas e incrementar la creatividad y } \\
\text { originalidad de sus publicaciones }\end{array}$ & 4 & 3,71 & 1,28 \\
\hline 9 & $\begin{array}{c}\text { Tener una mayor capacidad de provocación profesional, } \\
\text { desarrollando un periodismo más espectacular y } \\
\text { comprometido sin perjuicio del rigor }\end{array}$ & 4 & 2,57 & 1,68 \\
\hline 10 & $\begin{array}{c}\text { Ampliar los contenidos hacia otras áreas } \\
\text { de la comunicación y el marketing }\end{array}$ & 3 & 3 & 0,93 \\
\hline 11 & Fomentar una mayor especialización en los contenidos & 3 & 3 & 1,07 \\
\hline 12 & $\begin{array}{c}\text { Incentivar el patrocinio de seminarios y jornadas para } \\
\text { dar a conocer la publicación o incitar a la suscripción o lectura }\end{array}$ & 3 & 3,29 & 1,16 \\
\hline 13 & Centrarse más en la actualidad aumentando la periodicidad & 3 & 2,29 & 1,28 \\
\hline 14 & Promover su difusión internacional & 3 & 2,43 & 1,29 \\
\hline 15 & Aumentar la elaboración de contenidos a medida & 3 & 2,57 & 1,59 \\
\hline 16 & Adaptar su modelo a la telefonía móvil & 2 & 1,71 & 0,7 \\
\hline
\end{tabular}

Elaboración propia

En un segundo plano, los expertos creen que también son tendencias de futuro que deben ser enfatizadas las siguientes: incrementar el rigor en el contenido y el análisis de los temas, llevando a cabo una mayor profundización informativa (esto es así pese a que, de acuerdo con lo expuesto en la tabla II, los propios expertos defienden que ésta ya es una fortaleza o punto fuerte de las RPEPE); acercarse en mayor grado al mundo universitario; propiciar una mayor participación de los profesionales del sector; tener un contacto más estrecho y constante con la audiencia; potenciar el uso de nuevos formatos publicitarios en sus páginas e incrementar la creatividad y originalidad de sus publicaciones; y tener una mayor capacidad de provocación profesional, desarrollando un periodismo más espectacular y comprometido sin perjuicio del rigor (aunque en este último ítem hay un grado considerable de disenso entre los expertos).

$78\left|n^{\circ} 7\right|$ doxa.comunicación 
En un tercer escalón, son tendencias de futuro menos importantes ampliar los contenidos hacia otras áreas de la comunicación y el marketing, fomentar una mayor especialización, incentivar el patrocinio de jornadas para dar a conocer las revistas o incitar a la suscripción o lectura, centrarse más en la actualidad aumentando la periodicidad, promover su difusión internacional o aumentar la elaboración de contenidos a medida.

Para terminar, se pidió opinión a los expertos en lo referente al grado de saturación del mercado de las RPEPE y las posibilidades que existen para el surgimiento de nuevas cabeceras. En la primera ronda del proceso Delphi la respuesta fue casi unánime en el sentido de considerar el mercado como un mercado altamente saturado. En la segunda ronda, partiendo de ese consenso respecto al alto grado de saturación del mercado, se matizaron las respuestas (véase tabla VIII), pudiéndose concluir que para apenas un tercio de los expertos el mercado no admite ninguna cabecera más.

Tabla VIII. ¿Cuántas cabeceras más especializadas en publicidad admitiría el mercado español de estas revistas?

\begin{tabular}{|l|c|c|}
\hline Ítem & Frecuencia & Porcentaje \\
\hline $\begin{array}{l}\text { El mercado está saturado, pero hay lugar para nuevas } \\
\text { cabeceras siempre que sean títulos más especializados }\end{array}$ & 4 & 57,14 \\
\hline El mercado está saturado y no admite ninguna cabecera más & 2 & 28,57 \\
\hline $\begin{array}{l}\text { El mercado está saturado, pero es elástico y es posible } \\
\text { alguna cabecera más }\end{array}$ & 1 & 14,29 \\
\hline
\end{tabular}

Fuente: Elaboración propia

Ahora bien, los expertos que sí consideraron que en el mercado hay lugar para nuevos títulos afirmaron que esto puede suceder siempre y cuando se trate de cabeceras más especializadas. Así lo consideraron el 57\% de los expertos, mientras que sólo uno de los panelistas participantes defendió que el mercado es lo suficientemente elástico como para admitir alguna cabecera más aún cuando no sea más especializada que las actuales.

\section{Conclusiones}

Una vez expuestos los resultados más relevantes de la investigación que aquí presentamos, cerramos este artículo, a modo de síntesis, con algunas reflexiones acerca de las conclusiones más destacadas que se pue- 
den extraer de las mismas. Estas conclusiones nos permiten, por una parte, definir cuál es el estado actual de este sector y, por otra, esbozar cuáles son las principales líneas de actuación que marcarán el futuro de las publicaciones profesionales especializadas en publicidad.

En primer lugar y refiriéndonos al statu quo del mercado de las revistas objeto de estudio, los expertos coinciden unánimemente en señalar que se trata de un mercado saturado, aunque para la mayoría de los participantes en el cuestionario Delphi lo suficientemente flexible como para poder absorber nuevas cabeceras especializadas en nichos más específicos relacionados con el mundo publicitario.

El principal condicionante del crecimiento de este mercado es su decisiva dependencia de los ingresos por publicidad, lo cual es observado por algunos de los panelistas como una debilidad que llega incluso a determinar las rutinas productivas. Sirva como ejemplo la proliferación -o el "abuso", como se subraya en esta investigación- de especiales con los que se consigue atraer a un mayor número de anunciantes. Otro factor que limita el crecimiento de este sector es la estrategia de distribución, sustentada mayoritariamente en la suscripción, lo que genera una nueva debilidad subrayada por la mayoría de los expertos: la escasa visibilidad social de las RPEPE.

Entre las principales fortalezas de las cabeceras objeto de estudio cabe destacar su conocimiento del mundo publicitario fruto de una dilatada trayectoria, así como la buena imagen que estas publicaciones suscitan entre los profesionales del sector, la veteranía de los equipos humanos encargados de su realización y el saber hacer de los equipos de venta. La suma de todos estos factores ha conseguido que las RPEPE monopolicen la información sobre los contenidos especializados en publicidad en España, ya que, por el momento, no parecen existir en el mercado competidores relevantes ni en Internet ni en soporte papel que les disputen esta hegemonía. Sin embargo, los expertos reconocen tener en su punto de mira la evolución de los portales y websites sobre publicidad.

En cuanto a la utilización de las nuevas tecnologías de la información y la comunicación por las RPEPE, cabe destacar que a través de su posicionamiento en Internet los directivos persiguen básicamente tres objetivos: transmitir con mayor inmediatez las últimas noticias, actuar como hemeroteca de las informaciones publicadas y convertirse en plataforma-gancho para conseguir nuevos lectores para el papel.

Respecto a las conclusiones referidas al análisis prospectivo que se pretendía hacer en esta investigación de las RPEPE, podemos destacar una apuesta firme y consensuada por incluir contenidos de mayor profundidad que contribuyan al análisis y la interpretación de los cambios que se están produciendo en el sector de la publicidad sin que necesariamente tengan que tener una vertiente pragmática. Asimismo, los exper- 
tos consideran primordial conseguir una mayor visibilidad en el ámbito universitario a través de la difusión de información que pueda ser de interés para estudiantes y para aspirantes a la profesión. Por último, entre las tendencias de futuro, las cabeceras objeto de estudio aspiran a conseguir un mejor aprovechamiento de las nuevas tecnologías, principalmente Internet.

\section{Referencias bibliográficas}

Báez y Pérez de Tudela, J. (2007): Investigación cualitativa, Madrid: ESIC Editorial.

Berganza Conde, R. et al. (2005): Investigar en comunicación, Madrid: McGraw-Hill/Interamericana de España.

Gaitán Moya, J. A. y Piñuel Raigada, J. L. (1998): Técnicas de investigación de comunicación social, Madrid: Editorial Síntesis. Landeta, J. (2002): El método Delphi. Una técnica de previsión del futuro, Barcelona: Editorial Ariel.

Piñuel Raigada, J. L. y Gaitán Moya, J. A. (1999): Metodología general. Conocimiento científico e investigación en la comunicación social, Madrid: Editorial Síntesis.

Wimmer, R. D. y Dominick, J. R. (1996): La investigación científica de los medios de comunicación. Una introducción a sus métodos, Barcelona: Editorial Bosch. 
